\title{
A TOOL FOR MONITORING THE UNDER REPRESENTATION OF WOMEN IN CONFERENCES AND EVENTS
}

\begin{abstract}
The importance of participation in conferences and events is well known for members of the society. It is not only for the feedback and the personal experience, it is also about career development, building networks and increasing visibility. Nevertheless, women continue to be under-represented in these events and even more so in the most visible positions such as speaking roles. This paper presents the development of a tool based on performance indicators, which will allow monitoring and evaluating gender roles and inequalities in conferences and events in order to tackle the underrepresentation of women. The study identifies relevant perspectives (participation, organizational structure and attitudes) and designs specific lists of performance indicators for each of them. The tool is based on a combination of two multicriteria techniques, Analytic Hierarchy Process and Analytic Hierarchy Process Sort, and a qualitative analysis based on in-depth interviews and information gathered from a focus group. The use of the AHP multicriteria decision technique has allowed us to weight the indicators according to the opinion of several experts, and with them to be able to generate from these weightings composite indicators for each of the three dimensions. The most relevant indicators were for the participation dimension. Additionally, the tool developed has been applied to a conference which has been monitored in real time. The results are shown as a traffic light visualization approach, where red means bad performance, yellow average performance and green good performance, helping us to present the results for each indicator. Finally, proposals for improvement actions addressed to the red indicators are explained. The work carried out highlights the need to broaden the study of gender equality in conferences and events, not only regarding the participation but also the performance of different roles and functions.
\end{abstract}

Keywords: gender gap, women, AHP, AHP Sort, conferences, multi-criteria decision techniques.

\section{Introduction}

Gender gap is a concept that has to be analysed from several perspectives. Some of them are well known such as differential and asymmetric socialization and education, organizational culture, substantive representation, vertical segregation, work relations, visibility of women, gender perspective in research contents, gender expertise enhancement (Otero-Hermida and García-Melón, 2018) . Some others are still hidden or are not clearly publicized yet. The aim of this research is to identify all these relevant perspectives or dimensions, and to design specific lists of performance indicators for each of them. These indicators will allow the organizers of the conferences and events to monitor their performance according to each specific dimension. Performance indicators are supposed to shape behavior and International Symposium on the 1

WEB CONFERENCE Analytic Hierarchy Process

DEC. 3 - DEC. 6, 2020 
ISAHP Article: A Style Guide for Paper Proposals To Be Submitted to the International Symposium on the Analytic Hierarchy Process 2020, Web Conference.

practices in some desirable direction - in our case into a events practice 'with no gender gap’.

\section{Literature Review}

The study of the gender gap in science has gradually broadened to include different perspectives. Although there are many studies considering gender inequalities in academia, research in the participation in conferences and events remains limited (Débarre, Rode, \& Ugelvig, 2018; Nittrouer, Hebl, Ashburn-Nardo, Trump-Steele, \& Lane, 2017). Efforts have been made to highlight the critical underrepresentation of women ( Christal Morehouse, Alla Garcia, 2018). However, these previous studies can only be considered the first step towards a deeper understanding of the dimension of the gender gap.

Different research studies turn a spotlight on diverse aspects of women's participation in events but these studies have almost exclusively focused on one specific factor to analyse the gender differences in conferences, such as the proportion of female speakers (Nittrouer et al., 2017), the gender disparities in different leadership positions (Silva et al., 2019) or the influence of female organizing committee members in the selection of the speakers (Mehta et al., 2018) To the best of the authors' knowledge, no previous research has either considered different dimensions that can occur in the analysis of female participation in conferences or can be applied to different knowledge areas. Therefore, our new approach is needed in order to study gender disparity in different areas of knowledge and to develop an in-depth study of different roles and types of participation.

\section{Hypotheses/Objectives}

This paper addresses the need for a combinative study of different dimensions of the gender gap in conferences and events. We propose the development of a tool based on performance indicators, which will allow monitoring and evaluating gender roles and inequalities in events in order to tackle the underrepresentation of women. Since our main priority is to focus on mechanisms to enhance female representation, we propose indicators in a novel way using mixed methods with the support of the literature review and expert knowledge as a source of information and decision-making techniques.

Our goal is to identify all the relevant perspectives or dimensions related to the gender gap and to design specific lists of performance indicators for each of them.

\section{Research Design/Methodology}

For all the above reasons, gender gap should be treated as a multicriteria problem and therefore MCDA techniques are suitable for carrying out its monitorization. Thus, the complexity of this tool based on performance indicators will be tackled with the combination of two multicriteria techniques: Analytic Hierarchy Process (AHP) and Analytic Hierarchy Process Sort (AHP Sort). AHP allows us to assign weights to the indicators in order to develop composite indicators (1980). 
ISAHP Article: A Style Guide for Paper Proposals To Be Submitted to the International Symposium on the Analytic Hierarchy Process 2020, Web Conference.

Additionally, AHPSort classifies the conferences into the different levels of the criteria for their evaluation.

The following methodological approach has been proposed based on the use of MCDM techniques:

1. Selection of indicators (criteria) and relevant aspects (Literature review, interviews, participatory session).

2. Prioritization of the criteria by the experts. Use of AHP

3. Definition of measurement scales and threshold. Construction of composite indicators. Use of AHPSort

4. Monitoring of the events.

5. Discussion of the results

\section{Data/Model Analysis}

On the one hand, a thorough literature survey has been carried out in order to identify which dimensions (or criteria) should be considered when monitoring gender gap. On the other hand, interviews with experts have been carried out. After an in-depth analysis of these interviews, an additional list of indicators (criteria) of the gender gap has been obtained. Indicators (Criteria) have been arranged in a hierarchical structure and grouped in three relevant aspects: Participation, Organizational structure and Attitudes.

The monitoring and evaluation of the different events (alternatives) according to gender indicators has been carried out by means of AHPSort (Ishizaka, Pearman, \& Nemery, 2012). Since this is a sorting problem, thresholds (local limiting profiles), which indicate the minimum performance needed on each criterion to belong to a class (bad, average, good), have to be defined. To do that we had the help of the gender experts who also collaborated in the previous steps of the process.

\section{Limitations}

The most complex part is the work with experts when considering all the pairwise comparisons required by the AHP-Sort (Ishizaka et al., 2012). It will not always be easy to count on the collaboration of people linked to the organization of the event and with a historical vision of the evolution of the conference. That is why it is important to study the "terrain" beforehand and to have as many allies as possible within the conference organizing committee.

Moreover, owing to the need for physical presence in the sessions to record participation times, it has only been possible to measure this indicator in the sessions called keynotes. 
ISAHP Article: A Style Guide for Paper Proposals To Be Submitted to the International Symposium on the Analytic Hierarchy Process 2020, Web Conference.

\section{Conclusions}

With regard to the general methodology, we have been able to draw some conclusions. The use of multidisciplinary working groups has allowed us to have a more complete vision of the different approaches to the gender gap and thus to obtain a holistic and robust list of indicators.

In addition, the use of the AHP multi-criteria decision technique has allowed us to weight the indicators according to the opinion of several experts, again multidisciplinary, and with them to be able to generate from these weightings, composite indicators for each of the three dimensions. In this way, not only are results obtained with traffic light colours for the individual indicators, but also for each of the dimensions. Having each indicator and each dimension classified with a colour makes it much easier to see which indicators are performing well and above all which are not and need to be improved.

The assessment of each of the indicators has been carried out by applying the classification technique AHP-Sort. The use of this technique allows the classification into categories (traffic light colours) of the results obtained with the measurement of each indicator. Its application requires the expert knowledge of the people who set the limiting profiles that will serve as thresholds for each category. Therefore, this last stage of the methodology is not above criticism when it comes to predicting its replicability.

In general, we also want to emphasize that the creation of a tool to monitor academic events requires being able to use it independently of the discipline. Therefore, the applicability of our tool favours its use in any discipline. Likewise, the tool also allows us to compare results between different conferences.

\section{Key References}

Débarre, F., Rode, N. O., \& Ugelvig, L. V. (2018). Gender equity at scientific events. Evolution Letters, 2(3), 148-158. https://doi.org/10.1002/evl3.49

Ishizaka, A., Pearman, C., \& Nemery, P. (2012). AHPSort: An AHP-based method for sorting problems. International Journal of Production Research, 50(17), 47674784. https://doi.org/10.1080/00207543.2012.657966

Otero-Hermida, P., \& García-Melón, M. (2018). Gender equality indicators for research and innovation from a responsible perspective: The case of Spain. Sustainability, 10(9). https://doi.org/10.3390/su10092980

Saaty, T. L. (1980). The analytic hierarchy process: planning, priority setting, resources allocation. Mc. Graw Hill. https://doi.org/10.3414/ME10-01-0028 